\title{
Tri-allelic pattern at the TPOX locus: human identification in Brazil
}

\author{
Marek HF Ekert*, Marina L Mazanek, Carmélia S Miranda, Alessandro LN Santos and Rosana CF Silva \\ Laboratory of Forensic Genetics, Official Expertise of Alagoas, Brazil
}

\begin{abstract}
Introduction: Polymorphic short tandem repeats (STRs) are powerful genetic markers to distinguish individuals and still preferred for forensic applications and relationship testing. Tri-allelic pattern may have several causes, such as chimerism or chromosomal mutations.

Objective: This paper reports a tri-allelic pattern at the TPOX locus, which was encountered in our laboratory during analysis of an item from an attempted murder case.

Materials and methods: DNA extraction was the organic method with alcoholic precipitation. Real Time PCR quantified DNA samples, with the use of the reagent Quantifilier ${ }^{\circledR}$ TRIO DNA Quantification Kit. PCR analysis system approached by STRs IdentiFiler ${ }^{\circledR}$ and Capillary electrophoresis procedures were performed on the ABI 3500 Genetic Analyzer.

Results: This is the first report about tri-allelic pattern by forensic analysis in Brazil Northeast region. In the course of routine multiplex PCR-based forensic testing using microsatellite markers in the IdentiFiler ${ }^{\circledR}$ kit, we have found one case where the analysis pattern of one of the loci has yielded three peaks instead of the normal one or two.

Conclusions: The examination indicated the presence of tri-allelic pattern which indicates a standard tri-allelic type 2. Further, it is likely that powerful, high throughput genotyping methods will be applied in forensic testing in the future.
\end{abstract}

\section{Introduction}

Alleles at the TPOX STR locus have 6-14 different numbers of a four-nucleotide (AATG) repeat motif arranged in tandem [1]. Polymorphic short tandem repeats (STRs) are powerful genetic markers to distinguish individuals and still preferred for forensic applications and relationship testing [2]. These alleles can be observed as a two-banded pattern, as seen in a heterozygote, or a single banded pattern as seen in a homozygote. In very rare instances, a three banded pattern may be observed at a single locus in a multiplex STR profile, which is not a result of a mixture [3].

Although tri-allelic genotypes are generally rare, data presented on the STRBase website (http://www.cstl.nist.gov/biotech/strbase) indicate that short tandem repeats (STR) tri-allelic genotypes can be unusually frequent in the TPOX human STR locus. Therefore, TPOX polymorphism is widely used for paternity testing and personal identification, and is one of the FBI's CODIS STR loci (http://www.cstl. nist.gov/strbase/fbicore.htm) [4].

Tri-allelic pattern may have several causes, such as chimerism or chromosomal mutations. According to Clayton et al. [5], there are two types of tri-allelic pattern: type 1 , more frequent, where there is an imbalance in the size of the peaks, the sum of the peak intensity of the variants of the affected allele being equivalent to the intensity of the non-mutated allele, and type 2 , where the peaks have balanced intensity. Type 1 indicate somatic mutation of an allele at a heterozygous locus during the development of the individual, resulting in chimerism. Generally, the mutational event is the addition or loss of a repetitive unit. Tri-allelic patterns of type 2 indicate event of duplication located on the same chromosome or translocated or chromosomal aneuploidy (trisomy). When it comes to localized duplication, it is likely that the two alleles are inherited together because they will be strongly linked.

This paper reports a tri-allelic pattern at the TPOX locus, which was encountered in our laboratory during analysis of an item from an attempted murder case.

\section{Materials and methods}

The reference sample were subjected to DNA extraction using the organic method with alcoholic precipitation. Real Time PCR quantified DNA samples, with the use of the reagent Quantifilier TRIO DNA Quantification Kit (Life Technologies) and equipment QuantiStudio5 Real Time PCR System (Life Technologies). The extracted DNA were subjected to amplification by polymerase chain reaction (PCR), with the use of loci analysis system STRs IdentiFiler (Life Technologies) being the amplification products of these samples, genotyped for the loci D3S11358, VWA, D16S539, CSF1PO, TPOX, D8S1179, D21S11, D18S51, D19S433, TH01, FGA, D5S818, D13S317, D7S820, D2S1338 and amelogenin for the identification of the sexual gender. Capillary

${ }^{\star}$ Correspondence to: Marek HF Ekert, Laboratory of Forensic Genetics, Official Expertise of Alagoas, Brazil, Tel: + 55819.9795 3973, E-mail: marek.henryque@ gmail.com

Keywords: forensic DNA, tri-allelic pattern, TPOX, STR

Received: September 18, 2018; Accepted: September 28, 2018; Published: October 02,2018 
electrophoresis procedures were performed on the ABI 3500 Genetic Analyzer sequencing platform with POP-4 polymer and $36 \mathrm{~cm}$ capillary (Applied Biosystems).

\section{Results}

The study of nuclear DNA polymorphisms of autosomal chromosomes in tests carried out from samples collected, allowed to determine from the questioned samples, complete autosomal genetic profiles.

This is the first report about tri-allelic pattern by forensic analysis in Brazil Northeast region. In the course of routine multiplex PCRbased forensic testing using microsatellite markers in the IdentiFiler kit, we have found one case where the analysis pattern of one of the loci has yielded three peaks instead of the normal one or two (Figure 1).

Until April 2005 most of the tri-allelic pattern reported for the TPOX locus were type 2 [6]. Therefore, since TPOX occurs near the end of chromosome 2, it is more likely to duplicate for telomeres maintenance, in order to keep intact the chromosome end $[7,8]$.

Another interesting characteristic is that the great majority of the trialelias reported for the TPOX involves allele 10 and the most reported genotype (26 times) is $8 ; 10 ; 11$ [9], same as found in this research. Lane [10] in his work with populations showed that the extra allele in the trialelias of this locus was almost always 10, with apparently independent segregation, and that approximately twice as many relations to men showed this pattern, suggesting that the extra allele would be in the $\mathrm{X}$ chromosome. Despite the substantial frequency of the TPOX tri-allelic pattern, the nature of the third allele is still poorly understood.

Some general points concerning the emergence of triallelic patterns can be suggested based on previous reports and this article. The extensive study by Clayton et al. [5] on anomalous STR patterns for loci in the Identifiler kit (does not contain the TPOX locus) implies that triallelic patterns, although very rare, are to be expected in every traditional STR regions used in forensics. Crouse et al. [11] who report in 10,000 individuals a very high frequency of triallelic patterns at the TPOX locus (18 cases), whereas triallelic patterns at other tested loci. Internet database (http://www.cstl.nist.gov/biotech/strbase/), STR analyses may have a relatively high tendency to show triallelic patterns at the TPOX locus. The TPOX duplication is the most distal CNP found in chromosome $2 \mathrm{p}$. The closest CNP available in the public database (http://projects.tcag.ca/variation/) lies approximately $2 \mathrm{M}$ proximal from the duplication found by us and is located in the 2 p25.2 region [12].

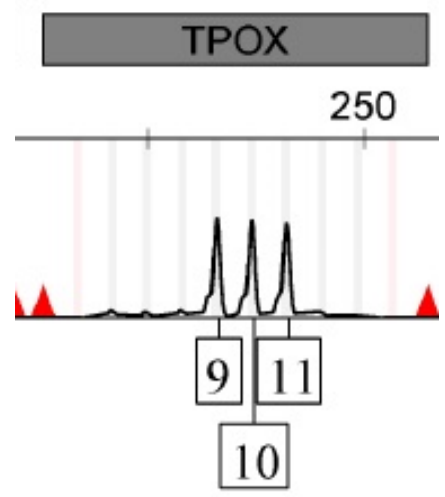

Figure 1. Part of electropherogram indicating the tri-allelic pattern in the genetic marker TPOX, using the IdentiFiler ${ }^{\circledR}$ kit (alleles 8,9 and 10)
The fact that a variety of bi-allelic combinations were transmitted to tri-allelic offspring suggests that the duplicated allele is not close to the TPOX locus as is the case with the triallelic TPOX genotype described by Lukka et al. [13]. This is further born out by the apparent linkage equilibrium between the 'duplication locus' and the main TPOX locus. The excess of tri-allelic females and the finding that tri-allelic males never transmit two alleles to their sons but always transmit two alleles to their daughters strongly suggests that the duplicated allele is on an $\mathrm{X}$ chromosome [10].

\section{Conclusions}

The examination indicated the presence of tri-allelic pattern which indicates a standard tri-allelic type 2 . Confirmation of this possibility comes from the fact alleles $8,9 \mathrm{e}, 10$ present in the electropherogram have a balanced signal. It suggests the genetic characterization of the population of Alagoas and other populations of the northeast region, in order to know possible population sub-structure. Further, it is likely that powerful, high throughput genotyping methods will be applied in forensic testing in the future.

\section{Acknowledgements}

Laboratório Forense of the Perícia Oficial de Alagoas for the collaboration and the support given to this study. All funding was provided by Government of the State of Alagoas, Brazil.

\section{References}

1. Anker R, Steinbrueck T, Donis-Keller H (1992) Tetranucleotide repeat polymorphism at the human thyroid peroxidase (hTPO) locus. Hum Mol Genet 1: 137. [Crossref]

2. Budowle B, Carracedo A, Brinkmann B (2003) Population studies on 17 STR loci routinely used in forensic analysis. Progress in Forensic Genetic 9: 71-74.

3. Butler JM (2001) Forensic DNA Typing. Academic Press.

4. Picanço JB, Raimann PE, Paskulin GA, Alvarez L, Amorim A, et al. (2014) Tri-allelic pattern at the TPOX locus: a familial study. Gene 535: 353-358. [Crossref]

5. Clayton TM, Guest JL, Urquhart AJ, Gill PD (2004) A genetic basis for anomalous band patterns encountered during DNA STR profiling. J Forensic Sci 49: 1207-1214. [Crossref]

6. Butler JM (2006) Genetics and genomics of core short tandem repeat loci used in human identity testing. J Forensic Sci 51: 253-265. [Crossref]

7. Chakhparonian M, Wellinger RJ (2003) Telomere maintenance and DNA replication: how closely are these two connected? Trends Genet 19: 439-446. [Crossref]

8. Louis EJ, Vershinin AV (2005) Chromosome ends: different sequences may provide conserved functions. Bioessays 27: 685-697. [Crossref]

9. STRBase. Available at: http://www.cstl.nist.gov/strbase/tri_tab.htm. (Accessed on 05/09/2018)

10. Lane AB (2008) The nature of tri-allelic TPOX genotypes in African populations Forensic Sci Int Genet 2: 134-137. [Crossref]

11. Crouse CA, Rogers S, Amiott E, Gibson S, Masibay A(1999) Analysis and interpretation of short tandem repeat microvariants and three-banded allele patterns using multiple allele detection systems. J Forensic Sci 44: 87-94. [Crossref]

12. Tuzun H, Sharp AJ, Bailey JA, Kaul R, Morrison VA, et al. (2005) Fine-scale structural variation of the human genome. Nat Genet 37: 727-732. [Crossref]

13. Lukka M, Tasa G, Ellonen P, Moilan K, Vassiljev V, et al. (2006) Triallelic patterns in STR loci used for paternity analysis: evidence for a duplication in chromosome 2 containing the TPOX STR locus. Forensic Sci Int 164: 3-9. [Crossref]

Copyright: C2018 Ekert MHF. This is an open-access article distributed under the terms of the Creative Commons Attribution License, which permits unrestricted use, distribution, and reproduction in any medium, provided the original author and source are credited. 